\title{
edmetic
}

\author{
Revista de Educación Mediática y TIC
}

\section{Hacia una implementación real y efectiva de la educación mediática en la ciudadanía}

\section{Towards a real and effective implementation of media education in citizenship}

Tras varias décadas desde el comienzo de las teorías y estudios sobre educomunicación, y en plena era digital y Sociedad del Conocimiento; el desarrollo de la educación mediática entre la ciudadanía se torna, hoy más que nunca, una exigencia inaplazable de cara a dotar a los ciudadanos de las herramientas necesarias para convivir y participar de manera proactiva en esta realidad digital e hipermedia que nos rodea.

En una sociedad en la que la dieta mediática determina y parcela el comportamiento del individuo durante las 24 horas del día, en la que el consumo de pantallas dictamina el flujo de información al que accedemos y la manera de relacionarnos con cualquier persona desde nuestro entorno más cercano; no podemos privar al ciudadano del derecho a recibir una educación sobre una realidad que marca decisivamente su forma de vivir y a la que posiblemente dedica un mayor número de horas que a la mayoría de parcelas y necesidades vitales que componen su vida cotidiana.

A día de hoy estamos en condiciones de afirmar que la educación mediática comprende la adquisición de toda una serie de competencias, entre las cuales se incluye necesariamente la digital, que tiene como objeto empoderar al ciudadano en el sentido crítico ante sus medios para que sea capaz de participar de las múltiples posibilidades que ofrece la realidad mediática en unas condiciones de libertad, integridad y conciencia social.

Desde principios de los años 2000, y acentuada por la irrupción de Internet y la generalización masiva de los medios digitales, existe una creciente 
aceptación tanto en la sociedad como en diversos organismos interestatales de la talla de ONU, UNESCO, Parlamento Europeo, etc., de la necesidad de caminar hacia el desarrollo efectivo de la competencia mediática por parte de toda la ciudadanía. La supervivencia y la participación libre en la realidad mediática que nos ofrece la Sociedad del Conocimiento pasa necesariamente por la adquisición de competencias que nos permitan: interactuar de manera eficaz con el creciente flujo de información a nuestro alcance, saber interpretar a todos los niveles los mensajes producidos por los múltiples medios y, yendo un paso más allá, ser capaces de crear de forma autónoma productos mediáticos con los que participar del universo comunicativo en condiciones de igualdad y con toda clase de garantías. El avance irrefrenable de los nuevos medios y sistemas de información y comunicación está originando a su vez nuevas formas de interrelación ciudadana que reinventan los métodos tradicionales de contacto y comunicación interpersonal.

En este sentido, las plataformas de redes sociales alcanzan cada día más peso en el porcentaje de interacción comunicativa que cientos de millones de personas realizan a diario en todo el mundo. Al mismo tiempo suponen una revolución, como nunca antes en la historia de la humanidad, del modo de contactar e intercambiar información con cualquier ser humano contemporáneo a nuestra época, con independencia del punto del planeta en el que se encuentre.

A pesar de todo ello, desde el ámbito académico y científico no cesan de llegar nuevas investigaciones y estudios que evidencian cómo una gran parte de la ciudadanía no tiene desarrolladas las competencias necesarias para aprovecharse de manera efectiva de las innumerables oportunidades que brinda el nuevo contexto digital ni tampoco para evitar los inconvenientes asociados a determinados tipos de consumo mediático. Riesgos que han aumentado considerablemente a la par que se ampliaba nuestro tiempo de vida online y que ya no consisten únicamente en no saber discernir la manipulación existente a la hora de consumir un producto mediático.

Así pues, en la actualidad asistimos al crecimiento incesante de problemas derivados del consumo digital que desde el sector de la educación 
mediática tenemos la responsabilidad de abordar y que de ahora en adelante deberán marcar el devenir de una parcela de los estudios encuadrados dentro de este ámbito académico. Nos referimos a los riesgos asociados al ámbito de la seguridad y la privacidad en Internet, a los problemas de veracidad informativa derivados de la saturación de fuentes y de la conocida como "sobreinformación", y por último al emergente campo de las adicciones digitales ligadas a la dependencia cada vez mayor de los nuevos dispositivos, principalmente smartphones y tablets.

Paralelamente, y como reafirman los organismos internacionales a través de sus recomendaciones y acuerdos en materia mediática, es en los gobiernos de los diferentes países donde recae la responsabilidad de crear la legislación y la coyuntura necesaria para garantizar la libertad de expresión y el libre acceso a la información y el conocimiento a escala global. En esa línea, el recientemente nombrado nuevo Secretario General de la ONU, António Guterres, ha afirmado en el día mundial de la libertad de prensa de 2017 que "necesitamos líderes que defiendan unos medios libres. Esto es crucial para contrarrestar la desinformación predominante. Necesitamos que todo el mundo apoye el derecho a la verdad". Sin embargo, a día de hoy continúan llegando noticias de acuerdos entre algunas de las principales potencias económicas en los que las libertades ciudadanas se encuentran recortadas, véase China, y grandes corporaciones de la industria digital para limitar el acceso libre a Internet y a determinadas fuentes de información. Alianzas selladas a cambio de beneficios económicos y otros intereses creados entre ambas partes.

Por todo ello, desde este monográfico también pretendemos hacer un llamamiento a favor de la libertad de prensa y del libre acceso a los medios digitales, como elementos indispensables para la rendición de cuentas que todo estado le debe a sus conciudadanos y como pilares sustanciales para el desarrollo humano en unas condiciones de igualdad, dignidad e integridad.

Para defender dicha convicción, resultan indispensables las investigaciones que tienen como objeto de estudio el desarrollo de la competencia digital de la ciudadanía así como la inclusión de la educación 
mediática. En este monográfico se pueden encontrar trabajos que las abordan desde diferentes ámbitos. Así, se recogen 14 artículos que giran en torno a la relación de la alfabetización mediática y la educación, amén de otros conceptos relacionados con estos, pero en los que también se analizan otros ámbitos como la comunicación o la política.

El primer artículo del volumen, Creación audiovisual para comprender Europa en Educación Secundaria. Aportaciones desde un proyecto Erasmust, de Blas Segovia Aguilar y Carmen Pavón, se adentra en el caso del programa europeo Erasmus Plus KA2, que tiene por finalidad la cooperación internacional, creando consorcios estratégicos para la innovación y el intercambio de buenas prácticas en el campo de la educación, formación y juventud.

En el segundo de los artículos, Competencia Digital: Uso y manejo de modelos 3D tridimensionales digitales e impresos en 3D, de José Luis Saorín, Cecile Meier, Jorge de la Torre-Cantrero, Carlos Carbonell-Carrera, Dámari Melián-Díaz y Alejandro Bonnet de León, profundiza de manera innovadora en un terreno emergente y tan novedoso como es el del modelado 3D y entornos virtuales tridimensionales (Realidad aumentada, virtual,...).

El tercer trabajo del monográfico, Caso de buenas prácticas en la formación en TIC y fomento de la competencia digital en la sociedad, y, especialmente, en los colectivos en riesgo de exclusión digital, de Nuria Hernández-León y Mario Miguel-Hernández, incide nuevamente en un ejemplo de buenas prácticas. Concretamente se trata de un caso destinado a la formación en TICS para fomentar la adquisición de competencia digital en la sociedad, haciendo especial énfasis en los colectivos que se encuentran en riesgo de exclusión digital.

El siguiente artículo, Rendimiento académico de los alumnos de secundaria que participan en el programa de aulas digitales, de Jeckson Enrique Loza Arenas, Verónica Salinas Urbina y Leonardo David Glasserman Morales, nos traslada al sector público colombiano para analizar el impacto de las aulas digitales en el rendimiento académico en alumnos de educación secundaria de una institución educativa.

La siguiente aportación del monográfico, Perspectiva histórica y claves 
actuales de la diversidad terminológica aplicada a la educación mediática, de Alejandro Buitrago Alonso, Agustín García Matilla y Alfonso Gutiérrez Martín, trata de arrojar luz en torno a un terreno tan cambiante en las últimas décadas, y tan discutido en ocasiones, como es el de la terminología más adecuada para denominar al ámbito académico de la educación en materia de comunicación.

El sexto artículo, Relaciones entre la prensa digital y los centros escolares de Educación Obligatoria, de Inmaculada Martín Herrera, tiene como objeto conocer a través de la percepción del profesorado el papel que desempeña la prensa digital en la Educación Obligatoria.

Se adentra nuevamente en el contexto iberoamericano el artículo La competencia TIC en el nuevo currículo peruano desde la perspectiva de la educación mediática, de Julio César Mateus y Cristobal Suárez Guerrero, pues aborda el nuevo currículo para la educación obligatoria que acaba de implementarse en Perú, el cual incluye una competencia específica sobre las Tecnologías de la Información y la Comunicación.

En el artículo Alfabetización mediática y cultura de la participación: retos de la ciudadanía digital en la Sociedad de la Información, Inés María Monreal Guerrero, José Luis Parejo y María de la O Cortón de las Heras abordan de forma teórica conceptos claves como son "alfabetización mediática". "ciudadanía digital" y "revolución informacional" y analizan los desafíos sociales a los que han dado lugar.

Beatriz Elena Marcano Lárez, Victoria Íñigo Mendoza y José Manuel Sánchez Ramírez, en su artículo titulado Percepción personal de una actividad de creación de contenido colaborativo de estudiantes del Máster de Formación del Profesorado se centran en la valoración de un proyecto colaborativo realizado en el Máster de Formación del Profesorado de Educación Secundaria para trabajar las competencias digitales de los docentes.

La alfabetización digital también es analizada desde otros ámbitos, no únicamente el educativo, como sucede en el trabajo La competencia digital de los políticos españoles en Twitter. El caso de las elecciones europeas de edmetic, 6(2), 2017, E-ISSN: 2254-0059; pp. 3-9. 
2014. Roberto Gelado Marcos y Fernando Bonete Vizcaíno analizan el uso que de Twitter que realizaron los líderes políticos españoles más significativos durante las Elecciones Europeas de 2014.

En Estrategias para la indagación continuada de trabajos académicos utilizando herramientas de Google. El caso de una investigación sobre infografías en la educación, Gonzalo Abio aborda un proceso de búsqueda bibliográfica sobre infografías en educación. Para ello, se emplean herramientas de Google que pueden ser útiles a la hora de realizar una investigación.

Beatriz Tarancón Álvaro es autora del artículo Implementación de un modelo de Entorno Personal de Aprendizaje en el Grado en Educación, en el que se analiza un estudio de caso centrado en un Entorno Personal de Aprendizaje (PLE) creado por el alumnado. En el trabajo se estudia la utilidad de los PLE así como el uso de la herramienta.

Bajo el título Es una locura vivir sin Facebook ni WhatsApp: la huella tecnológica en el docente en formación, Miriam Sonlleva Velasco, Alba Torrego González y Suyapa Martínez Scott se centran en la investigación biográfica- narrativa. Las autoras analizan tecnobiografías escritas por alumnado de primer curso de Grado de Maestro para conocer sus vivencias tecnológicas en contextos formales e informales y reflexionan sobre la necesidad de incorporar en la formación inicial del profesorado contenidos crítico- reflexivos relacionados con las TIC.

Cierra este monográfico un trabajo enviado desde México por Abel Grijalvo Verdugo y María Luisa Urrea Zazueta que lleva por título Evaluación de la competencia digital en estudiantes de comunicación. El caso de una universidad mexicana. En él, los autores analizan los niveles de competencia digital de los estudiantes en comunicación de una universidad mexicana, centrándose en la información y alfabetización informacional y en la creación de contenidos digitales.

Como hemos podido comprobar, el presente monográfico sobre "Educación Mediática y Competencia Digital" de la Revista Edmetic cuenta con un privilegiado compendio de artículos que reflexionan acerca de este campo de estudios heredero de la mejor tradición educomunicativa. Aquellos 
interesados en la materia que se adentren en su lectura podrán descubrir toda una serie de investigaciones y buenas prácticas llevadas a cabo de forma reciente en el ámbito académico de la educación mediática y que, en su conjunto, plantean propuestas de actuación de cara al futuro más inmediato de la formación en medios. Una apuesta editorial y científica que lleva aparejado un fin primordial: fomentar la implantación real y efectiva de la educación mediática y la competencia digital en la sociedad a todos sus niveles.

\author{
Alba Torrego \\ correo@uva.es \\ Alejandro Buitrago \\ correo@uva.es \\ Universidad de Valladolid
}

edmetic, 6(2), 2017, E-ISSN: 2254-0059; pp. 3-9.

(c) edmetic, Revista de Educación Mediática y TIC 